\title{
Antibiotic resistance assessment in bacteria isolated in migratory Passeriformes transiting through the Metaponto territory (Basilicata, Italy)
}

\author{
Maria Foti ${ }^{*}$ (D) Antonietta Mascetti ${ }^{1}$, Vittorio Fisichella ${ }^{1}$, Egidio Fulco ${ }^{2}$, Bianca Maria Orlandella ${ }^{1}$ \\ and Francesco Lo Piccolo ${ }^{1}$
}

\begin{abstract}
Background: Wild birds are considered to be reservoirs of human enteric pathogens and vectors of antimicrobial resistance dissemination in the environment. During their annual migration, they play a potential role in the epidemiology of human associated zoonoses. The aim of this study was to investigate the frequency of isolation and antimicrobial susceptibility profiles of microorganisms found in the cloaca of common European passerines.

Methods: One hundred and twenty-one cloacal swabs were collected during a monitoring program of migratory birds in the Forest Reserve for Protection "Metaponto" (Basilicata, Italy). All samples were cultured using standard bacteriological methods and antibiotic susceptibility testing (agar disk diffusion test) of isolated strains was performed.

Results: The bacteriological analysis produced 122 strains belonging to 18 different species. The most commonly isolated species were Enterobacter cloacae and Providencia rettgeri (21 strains, 17.2\%). Potentially pathogenic species including Klebsiella pneumoniae, Serratia marcescens and Pseudomonas spp. have also been identified. Isolates showed significant frequencies of antimicrobial resistance. The highest frequency of resistance was observed against amoxicillin $(n=79,64.8 \%)$; ampicillin ( $n=77,63.1 \%)$; rifampicin $(n=75,61.5 \%)$; amoxicillin-clavulanic acid $(n=66,54.1 \%)$. Thirty-one strains (25.4\%) showed resistance to imipenem and 8 (6.6\%) to meropenem.

Conclusions: Migratory birds play an important role in the ecology, circulation and dissemination of potentially pathogenic antimicrobial resistant organisms. They can therefore be considered sentinel species and environmental health indicators. Our results suggest that the integration of epidemiological surveillance networks during ringing campaigns of wild species can be an effective tool to study this phenomenon.
\end{abstract}

Keywords: Passeriformes, Cloacal swabs, Bacteriological test, Antimicrobial resistance

\section{Background}

Several studies have shown that migratory wild birds play an important role in the ecology, circulation and dissemination of enteric human pathogens such as Campylobacter, Salmonella, toxin-producing Escherichia coli and

*Correspondence: malinvet@unime.it

1 Department of Veterinary Sciences, University of Messina, Polo

Universitario dell'Annunziata, 98168 Messina, Italy

Full list of author information is available at the end of the article antimicrobial resistant organisms (Reed et al. 2003; Abulreesh et al. 2007; Foti et al. 2011; Magda et al. 2013).

Although these birds come rarely in contact with antimicrobial agents, they could serve as reservoirs and potential disseminators of resistant bacteria in the environment through fecal depositions (Guenther et al. 2010; Jarhult et al. 2013; Shobrak and Abo-Amer 2015). Resistant bacteria of human and veterinary origin are believed to be transmitted to wild birds through contaminated food or water (Abulreesh et al. 2007; Bonnedahl et al. 2009; Guenther et al. 2010; Radhouani et al. 2012). 
Residues of antibiotics and bacteria carrying antibiotic resistance may be introduced into the environment due to the spread of manure from medicated livestock and urban effluents into agricultural land (Blanco et al. 2009). At rest sites, birds of different species often congregate and the horizontal transmission of pathogens occurs due to interindividual and interspecies contact (Hubàlek 2004), including interaction with sedentary birds.

Furthermore, heavy stress and immunosuppression related to migration could promote the onset of infectious diseases and the spread of infectious agents. Other factors contributing to the prevalence of resistant bacterial strains in wild birds are the environmental contamination, the presence of livestock and human density (Skurnik et al. 2006; Allen et al. 2010). Several studies have shown a wide spread of antibiotic resistant enterobacteria in bird populations sympatric to areas inhabited by people and areas with a high density of livestock (Camarda et al. 2006; Literak et al. 2010; Elmberg et al. 2017).

Most of the information regarding bacterial enteropathogens in wild birds stems from the application of traditional microbiological techniques adapted to the study of those species that are most likely to affect human health. In a previous research carried out by Giacopello et al. (2016) the most frequently diffused resistances among Enterobacteriaceae isolated from passerines in a wildlife rescue centre in Sicily were found to be trimethoprim/sulfamethoxazole (100\%), streptomycin (56.2\%), amoxicillin/clavulanic acid (62.5\%), ampicillin (50\%) and tetracycline (31.2\%) (Giacopello et al. 2016). Strains of Escherichia spp. isolated from migratory wild birds from different areas of Saudi Arabia displayed resistance to chloramphenicol (100\%), oxytetracycline (100\%), ciprofloxacin (87.5\%), ampicillin (75\%), cefaclor (62.5\%), cephalexin (62.5\%) and amoxicillin (50\%) (Shobrak and Abo-Amer 2015). Guenther et al. (2010) evaluated the susceptibility of 187 Escherichia coli isolates from 226 European wild birds (117 of which belonging to the order Passeriformes) to different antimicrobials and found resistance to ampicillin, cephalotin, tetracycline and neomycin in $60,46.6,46.6$ and $33.3 \%$ of the isolates, respectively (Guenther et al. 2010).

In 2010, the Territorial Office for Biodiversity of the Italian Forestry Corps had set up a monitoring program in the Forest Reserve for Protection "Metaponto", located in the Matera province (Italy), with the aim of studying the migratory avifauna along the Ionian Basilicata Coast. The research activities are becoming especially intense during autumn, when several species of migratory passerines (especially intrapaleartic) stop to rest in extended formations of Mediterranean maquis and in the remaining retrodunal wetlands.

During the tracking season a population of migratory birds has been subjected to health evaluation through various laboratory tests. The study aimed to acquire new data about the bacterial flora of migratory populations passing through Italy by focusing on the isolation of Enterobacteriaceae and by recording the eventual presence of pathogens in all captured specimens. Furthermore, the antimicrobial susceptibility of the isolated strains was tested in order to highlight the possible spread of the antimicrobial resistance in animals that, surely, have never received therapeutic protocols and can therefore be considered environmental sentinels.

\section{Methods \\ Sampling}

The catches were made near the mouth of the river Bradano using $276 \mathrm{~m}$ of mist-net $12 \times 2$, kept open from dawn to dusk and monitored every hour.

In 121 subjects belonging to the Order Passeriformes (Table 1) cloacal swabs were obtained by inserting a sterile culture swab impregnated with buffered peptone

Table 1 Classification of sampled avian species

\begin{tabular}{lllr}
\hline Family & Common name & Scientific name & Number of samples \\
\hline Emberizidae & Reed Bunting & Emberiza schoeniclus (Linnaeus, 1758) & 1 \\
Fringillidae & Chaffinch & Fringilla coelebs (Linnaeus, 1758) & 2 \\
Sylviidae & Chiffchaff & Phylloscopus collybita (Vieillot, 1817) & 6 \\
& Firecrest & Regulus ignicapilla (Temminck, 1820) & 1 \\
& Eurasian Blackcap & Sylvia atricapilla (Linnaeus, 1758) & 39 \\
Turdidae & European Robin & Erithacus rubecula (Linnaeus, 1758) & 68 \\
& Blackbird & Turdus merula (Linnaeus, 1758) & 2 \\
Total & Song Thrush & Turdus philomelos (Brehm, 1831) & 2 \\
\hline
\end{tabular}


water (Oxoid, Basingstoke, UK) into the cloaca and gently rotating the tip against the mucosa.

\section{Laboratory procedures Bacterial isolation and identification}

Samples were transported in refrigeration conditions to the laboratory of Microbiology of the Department of Veterinary Sciences, University of Messina (Italy) and then submitted to standard bacteriological examination for detection of Enterobacteriaceae. After an enrichment in buffered peptone water, the samples were streaked into MacConkey Agar plates (Oxoid, Basingstoke, Hampshire, UK) using sterile loops. Isolates were subcultured in Blood Agar plates for identification by mass spectrometry MALDI-TOF (matrix assisted laser desorption/ionisation-time of flight mass spectrometry). The isolated colonies were seeded in a 48-well metal plate with disposable loops, using as a reference strain Escherichia coli ATCC 8739. The results were analyzed with the VITEK MS system (bioMérieux SA, Marcy l'Etoile, France), using the software Axima (Shimadzu Kyoto, Japan)-SARAMIS database (Spectral ARchive And Microbial Identification System) (AnagnosTec, Berlin, Germany).

\section{Antimicrobial susceptibility testing}

Antimicrobial susceptibility testing of the bacterial isolates was performed by disk diffusion method (Bauer et al. 1966) on Mueller-Hinton agar (Oxoid, Basingstoke, UK) in accordance to international standards (CLSI 2013). Susceptibility to 18 antimicrobial agents belonging to 9 antibiotics classes was evaluated: amikacin (AK, $30 \mu \mathrm{g}$ ), amoxicillin (AML, $30 \mu \mathrm{g}$ ), amoxicillin/clavulanic acid (AUG, $30 \mu \mathrm{g}$ ), ampicillin (AMP, $10 \mu \mathrm{g}$ ), aztreonam (ATM, $30 \mu \mathrm{g}$ ), cefotaxime (CTX, $30 \mu \mathrm{g}$ ), cefotaxime/clavulanic acid (CTL, $40 \mu \mathrm{g}$ ), ceftazidime (CAZ, $30 \mu \mathrm{g})$, ceftazidime/clavulanic acid (CAL, $40 \mu \mathrm{g})$, ciprofloxacin (CIP, $5 \mu \mathrm{g})$, enrofloxacin (ENR, $5 \mu \mathrm{g})$, gentamicin $(\mathrm{CN}, 10 \mu \mathrm{g})$, imipenem (IMI, $10 \mu \mathrm{g}$ ), meropenem (MEM, $10 \mu \mathrm{g}$ ), rifampicin (RD, $30 \mu \mathrm{g})$, tetracycline ( $\mathrm{TE}, 30 \mu \mathrm{g})$, tobramycin (TOB, $10 \mu \mathrm{g}$ ), trimethoprim/sulfamethoxazole (SXT, $50 \mu \mathrm{g}$ ) (Liofilchem, Teramo, IT). Isolates were considered resistant or susceptible according to the manufacturer's instructions based on CLSI guidelines (Liofilchem ${ }^{\circledR}$ 2016). Isolates showing intermediate susceptibility were considered as resistant. Strains were considered multidrug resistant (MDR) when showing resistance to three or more antimicrobial classes (Schwarz et al. 2010).

\section{Statistical analysis}

The statistical analysis of the results was made using the $z$-test. Differences were considered significant at values of $p<0.05$.

\section{Results}

Bacterial isolation and identification

One hundred and twenty-two strains were isolated. Of these 114 belonged to 10 different genera and 8 were unidentified (Table 2).

The most commonly isolated species were Providencia rettgeri (23 strains, 18.9\%), Enterobacter cloacae (21 strains, 17.2\%) and Leclercia adecarboxylata (16 strains, $14.7 \%$ ). Potentially pathogenic species including Klebsiella pneumoniae, Serratia marcescens and Pseudomonas spp. have also been identified.

There were no significant differences in the frequencies of microorganism among the most common bird species (Table 3).

\section{Antimicrobial susceptibility testing}

Isolates displayed significant frequencies of antibiotic resistance (Table 4).

Twenty-four strains showed resistance to more than $50 \%$ of the tested molecules. The resistance patterns varied from one to sixteen of the antibiotics tested. The resistance to amoxicillin $(n=79,64.8 \%)$ was the most frequent, followed by ampicillin $(n=77,63.1 \%)$, rifampicin $(n=75,61.5 \%)$ and amoxicillin-clavulanic acid $(n=66,54.1 \%)$.

\section{Table 2 Identification of bacterial species}

\begin{tabular}{lc}
\hline Isolated species & Number of strains \\
\hline Aeromonas spp. & 1 \\
Aeromonas punctata & 3 \\
Citrobacter spp. & 6 \\
Citrobacter braaki & 1 \\
Citrobacter farneri & 2 \\
Citrobacter freundii & 4 \\
Enterobacter spp. & 4 \\
Enterobacter asburiae & 2 \\
Enterobactercancerogenus & 8 \\
Enterobactercloacae & 21 \\
Enterobacter cowanii & 1 \\
Hafnia alvei & 4 \\
Klebsiella oxytoca & 2 \\
Klebsiella pneumoniae & 2 \\
Leclercia spp. & 2 \\
Leclercia adecarboxylata & 16 \\
Proteus mirabilis & 1 \\
Providencia rettgeri & 23 \\
Pseudomonas putida & 2 \\
Pseudomonas stutzeri & 22 \\
Serratia liquefaciens & 122 \\
Serratia marcescens & 1 \\
Unidentified & 6 \\
Total & 2 \\
\hline &
\end{tabular}




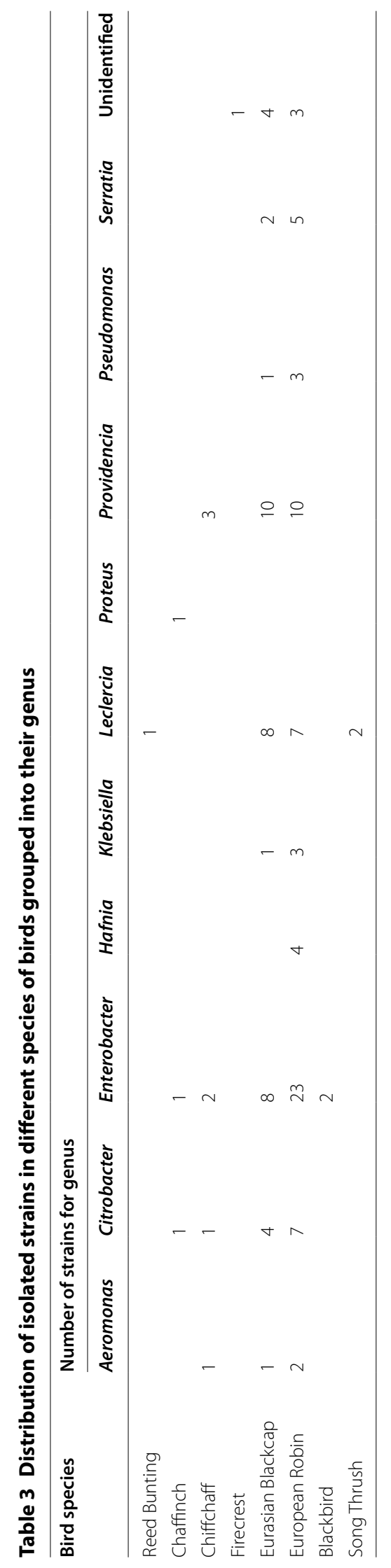


Table 4 Number of resistant strains for single molecules and single bacterial species

\begin{tabular}{|c|c|c|c|}
\hline Class & Antibiotics & Bacterial species $^{a}$ & Total number of resistant strains \\
\hline \multirow[t]{3}{*}{ Aminoglycosides } & Amikacin & $\begin{array}{l}\text { Citrobacter farneri (1) } \\
\text { Citrobacter freundii (3) } \\
\text { Enterobacter asburiae (1) } \\
\text { Enterobacter cancerogenus (2) } \\
\text { Enterobacter cloacae (6) } \\
\text { Hafnia alvei (1) } \\
\text { Klebsiella pneumoniae (1) } \\
\text { Leclercia adecarboxylata (2) } \\
\text { Providencia rettgeri (6) } \\
\text { Pseudomonas putida (1) } \\
\text { Serratia marcescens (1) }\end{array}$ & $25(20.5 \%)$ \\
\hline & Gentamicin & $\begin{array}{l}\text { Citrobacter freundii (3) } \\
\text { Citrobacter spp. (2) } \\
\text { Enterobacter asburiae (1) } \\
\text { Enterobacter cancerogenus (2) } \\
\text { Enterobacter cloacae (6) } \\
\text { Klebsiella oxytoca (1) } \\
\text { Klebsiella pneumoniae (1) } \\
\text { Leclercia adecarboxylata (2) } \\
\text { Providencia rettgeri (1) } \\
\text { Pseudomonas putida (1) }\end{array}$ & $20(16.4 \%)$ \\
\hline & Tobramicina & $\begin{array}{l}\text { Citrobacter freundii (2) } \\
\text { Citrobacter spp. (1) } \\
\text { Enterobacter cancerogenus (3) } \\
\text { Enterobacter cloacae (7) } \\
\text { Klebsiella oxytoca (1) } \\
\text { Klebsiella pnemoniae (1) } \\
\text { Leclercia adecarboxilata (3) } \\
\text { Providencia rettgeri (3) } \\
\text { Serratia marcescens (1) }\end{array}$ & $22(18 \%)$ \\
\hline \multirow[t]{4}{*}{ Cephalosporins } & Cefotaxime & $\begin{array}{l}\text { Citrobacter freundii (1) } \\
\text { Enterobacter asburiae (1) } \\
\text { Enterobacter cancerogenus (3) } \\
\text { Enterobacter cloacae (6) } \\
\text { Klebsiella pneumoniae (1) } \\
\text { Providencia rettgeri (3) } \\
\text { Pseudomonas putida (1) }\end{array}$ & $16(13.1 \%)$ \\
\hline & Cefotaxime-clavulanic acid & $\begin{array}{l}\text { Citrobacter freundii (2) } \\
\text { Enterobacter asburiae (1) } \\
\text { Enterobacter cancerogenus (2) } \\
\text { Enterobacter cloacae (6) } \\
\text { Klebsiella pneumoniae (1) } \\
\text { Providencia rettgeri (3) } \\
\text { Pseudomonas putida (1) }\end{array}$ & $16(13.1 \%)$ \\
\hline & Ceftazidime & $\begin{array}{l}\text { Citrobacter farneri (1) } \\
\text { Citrobacter freundii (2) } \\
\text { Citrobacter spp. (1) } \\
\text { Enterobacter asburiae (1) } \\
\text { Enterobacter cancerogenus (3) } \\
\text { Enterobacter cloacae (8) } \\
\text { Klebsiella pneumoniae (1) } \\
\text { Leclercia adecarboxylata (2) } \\
\text { Providencia rettgeri (4) } \\
\text { Pseudomonas putida (1) }\end{array}$ & $24(19.7 \%)$ \\
\hline & Ceftazidime-clavulanic acid & $\begin{array}{l}\text { Citrobacter farneri (1) } \\
\text { Citrobacter freundii (2) } \\
\text { Citrobacter spp. (1) } \\
\text { Enterobacter asburiae (1) } \\
\text { Enterobacter cancerogenus (3) } \\
\text { Enterobacter cloacae (8) } \\
\text { Klebsiella pneumoniae (1) } \\
\text { Providencia rettgeri (7) } \\
\text { Pseudomonas putida (1) }\end{array}$ & $25(20.5 \%)$ \\
\hline
\end{tabular}


Table 4 continued

\begin{tabular}{|c|c|c|c|}
\hline Class & Antibiotics & Bacterial species $^{a}$ & Total number of resistant strains \\
\hline \multirow[t]{2}{*}{ Carbapenems } & Imipenem & $\begin{array}{l}\text { Citrobacter farneri (1) } \\
\text { Citrobacter freundii (2) } \\
\text { Citrobacter spp. (1) } \\
\text { Enterobacter asburiae (2) } \\
\text { Enterobacter cancerogenus (4) } \\
\text { Enterobacter cloacae (9) } \\
\text { Klebsiella pneumoniae (1) } \\
\text { Leclercia adecarboxylata (2) } \\
\text { Providencia rettgeri (7) } \\
\text { Pseudomonas putida (1) } \\
\text { Serratia marcescens (1) }\end{array}$ & 31 (25.4\%) \\
\hline & Meropenem & $\begin{array}{l}\text { Enterobacter cancerogenus (2) } \\
\text { Enterobacter cloacae (1) } \\
\text { Leclercia adecarboxylata (2) } \\
\text { Providencia rettgeri (2) } \\
\text { Pseudomonas putida (1) }\end{array}$ & $8(6.6 \%)$ \\
\hline \multirow[t]{2}{*}{ Fluoroquinolones } & Ciprofloxacin & $\begin{array}{l}\text { Citrobacter freundii (2) } \\
\text { Citrobacter spp. (2) } \\
\text { Enterobacter cancerogenus (1) } \\
\text { Enterobacter cloacae (6) } \\
\text { Klebsiella pneumoniae (1) }\end{array}$ & $12(9.8 \%)$ \\
\hline & Enrofloxacin & $\begin{array}{l}\text { Citrobacter freundii (2) } \\
\text { Citrobacter spp. (2) } \\
\text { Enterobacter cancerogenus (1) } \\
\text { Enterobacter cloacae (1) } \\
\text { Klebsiella oxytoca (1) } \\
\text { Klebsiella pneumoniae (1) } \\
\text { Leclercia adecarboxylata (1) } \\
\text { Providencia rettgeri (2) } \\
\text { Pseudomonas putida (1) } \\
\text { Serratia marcescens (2) }\end{array}$ & $14(11.5 \%)$ \\
\hline Monobactams & Aztreonam & $\begin{array}{l}\text { Citrobacter farneri (1) } \\
\text { Citrobacter freundii (1) } \\
\text { Citrobacter spp. (1) } \\
\text { Enterobacter asburiae (1) } \\
\text { Enterobacter cancerogenus (4) } \\
\text { Enterobacter cloacae (7) } \\
\text { Klebsiella oxytoca (1) } \\
\text { Providencia rettgeri (5) } \\
\text { Pseudomonas putida (1) } \\
\text { Pseudomonas stutzeri (1) } \\
\text { Serratia marcescens (1) } \\
\text { Non-identified (1) }\end{array}$ & $25(20.5 \%)$ \\
\hline Penicillins & Amoxicillin & $\begin{array}{l}\text { Aeromonas punctata (1) } \\
\text { Aeromonas spp. (1) } \\
\text { Citrobacter farneri (2) } \\
\text { Citrobacter freundii (4) } \\
\text { Citrobacter spp. (3) } \\
\text { Enterobacter asburiae (2) } \\
\text { Enterobacter cancerogenus (8) } \\
\text { Enterobacter cloacae (18) } \\
\text { Enterobacter cowanii (1) } \\
\text { Enterobacter spp. (4) } \\
\text { Hafnia alvei (1) } \\
\text { Klebsiella oxytoca (2) } \\
\text { Klebsiella pneumoniae (2) } \\
\text { Leclercia adecarboxylata (6) } \\
\text { Proteus mirabilis (1) } \\
\text { Providencia rettgeri (12) } \\
\text { Pseudomonas putida (1) } \\
\text { Serratia marcescens (5) } \\
\text { Non-identified (5) }\end{array}$ & 79 (64.8\%) \\
\hline
\end{tabular}


Table 4 continued

\begin{tabular}{|c|c|c|c|}
\hline Class & Antibiotics & Bacterial species $^{a}$ & Total number of resistant strains \\
\hline & Amoxicillin-clavulanic acid & $\begin{array}{l}\text { Aeromonas punctata (1) } \\
\text { Aeromonas spp. (1) } \\
\text { Citrobacter farneri (2) } \\
\text { Citrobacter freundii (4) } \\
\text { Citrobacter spp. (3) } \\
\text { Enterobacter asburiae (2) } \\
\text { Enterobacter cancerogenus (8) } \\
\text { Enterobacter cloacae (17) } \\
\text { Enterobacter spp. (4) } \\
\text { Hafnia alvei (1) } \\
\text { Klebsiella pneumoniae (1) } \\
\text { Leclercia adecarboxylata (3) } \\
\text { Providencia rettgeri (12) } \\
\text { Pseudomonas putida (1) } \\
\text { Serratia marcescens (3) } \\
\text { Non-identified (3) }\end{array}$ & $66(54.1 \%)$ \\
\hline & Ampicillin & $\begin{array}{l}\text { Aeromonas punctata (1) } \\
\text { Aeromonas spp. (1) } \\
\text { Citrobacter farneri (1) } \\
\text { Citrobacter freundii (4) } \\
\text { Citrobacter spp. (2) } \\
\text { Enterobacter asburiae (1) } \\
\text { Enterobacter cancerogenus (8) } \\
\text { Enterobacter cloacae (18) } \\
\text { Enterobacter cowanii (1) } \\
\text { Enterobacter spp. (4) } \\
\text { Hafnia alvei (1) } \\
\text { Klebsiella oxytoca (2) } \\
\text { Klebsiella pneumoniae (2) } \\
\text { Leclercia adecarboxylata (5) } \\
\text { Proteus mirabilis (1) } \\
\text { Providencia rettgeri (15) } \\
\text { Pseudomonas putida (1) } \\
\text { Pseudomonas stutzeri (1) } \\
\text { Serratia marcescens (4) } \\
\text { Non-identified (4) }\end{array}$ & $77(63.1 \%)$ \\
\hline Rifamycins & Rifampicin & $\begin{array}{l}\text { Aeromonas punctata (1) } \\
\text { Citrobacter farneri (2) } \\
\text { Citrobacter freundii (4) } \\
\text { Citrobacter spp. (5) } \\
\text { Enterobacter asburiae (2) } \\
\text { Enterobacter cancerogenus (7) } \\
\text { Enterobacter cloacae (16) } \\
\text { Enterobacter cowanii (1) } \\
\text { Enterobacter spp. (4) } \\
\text { Hafnia alvei (3) } \\
\text { Klebsiella oxytoca (1) } \\
\text { Klebsiella pneumoniae (2) } \\
\text { Leclercia adecarboxylata (10) } \\
\text { Leclercia spp. (1) } \\
\text { Providencia rettgeri (9) } \\
\text { Pseudomonas putida (1) } \\
\text { Pseudomonas stutzeri (1) } \\
\text { Serratia marcescens (4) } \\
\text { Non-identified (2) }\end{array}$ & $76(61.5 \%)$ \\
\hline Sulfonamides & Trimethoprim/sulfamethoxazole & $\begin{array}{l}\text { Citrobacter freundii (1) } \\
\text { Enterobacter cloacae (2) } \\
\text { Leclercia adecarboxylata (1) } \\
\text { Providencia rettgeri (7) } \\
\text { Pseudomonas stutzeri (1) } \\
\text { Non-identified (3) }\end{array}$ & $15(12.3 \%)$ \\
\hline
\end{tabular}


Table 4 continued

\begin{tabular}{|c|c|c|c|}
\hline Class & Antibiotics & Bacterial species $^{a}$ & Total number of resistant strains \\
\hline Tetracyclines & Tetraciclina & $\begin{array}{l}\text { Citrobacter freundii (2) } \\
\text { Citrobacter spp. (1) } \\
\text { Enterobacter asburiae (1) } \\
\text { Enterobacter cancerogenus (3) } \\
\text { Enterobacter cloacae (8) } \\
\text { Klebsiella oxytoca (1) } \\
\text { Leclercia adecarboxylata (3) } \\
\text { Proteus mirabilis (1) } \\
\text { Providencia rettgeri (21) } \\
\text { Pseudomonas putida (1) } \\
\text { Serratia marcescens (4) } \\
\text { Non-identified (2) }\end{array}$ & $48(39.3 \%)$ \\
\hline
\end{tabular}

a In the brackets are the numbers of resistant strains

Thirty-one strains (25.4\%) showed resistance to imipenem and $8(6.6 \%)$ to meropenem. Multidrug resistance occurred in 35/122 strains (28.7\%). Among strains resistant to the cephalosporins none showed a phenotypic ESBL profile. Nine strains were susceptible to all tested molecules. Some bacterial species have shown resistance against numerous molecules (Table 5).

Particularly, Enterobacter cloacae has shown resistance to 18 molecules (100\%); Enterobacter cancerogenus, Citrobacter freundii and Providencia rettgeri to 17 molecules (94.4\%); Pseudomonas putida to 15 molecules (83.3\%); Klebsiella pneumoniae to 14 molecules (77.8\%); Citrobacter spp., Enterobacter asburiae and Leclercia adecarboxylata to 13 molecules (72.2\%). There were no significant differences in the percentage of resistant bacteria among the different bird species (Table 6).

\section{Discussion}

Bacteriological analysis led to the isolation of a wide range of bacterial species. Several of the isolated bacteria, such as K. pneumoniae, Enterobacter spp., Proteus spp., Providencia spp. and Citrobacter spp., are known to cause diseases in avian species, as well as in mammals and humans (Reslinski et al. 2005; Pindi et al. 2013).

Unlike previous studies on wild birds, no strains of Salmonella spp. and Escherichia coli have been isolated (Hubàlek 2004; Benskin et al. 2009; Guenther et al. 2010; Matias et al. 2016). This result might partially be explained because of diet, as these species were most commonly found in surveys of omnivorous birds as well as carnivorous birds (Bangert et al. 1988), whereas graminivorous birds, such as many passerines, had much lower prevalence (Glunder 1981; Brittingham et al. 1988; Steele et al. 2005). Antimicrobial susceptibility testing revealed a wide spread of strains resistant to some of the molecules tested. The percentage of resistance to penicillins is consistent with the results obtained by other authors (Guenther et al. 2010; Shobrak and Abo-Amer 2015; Giacopello et al. 2016).

The intake of water polluted with faeces or human waste and the acquisition via food seem to be the sources of transmission of resistant bacteria of human and veterinary origin to wild birds (Reed et al. 2003; Pindi et al. 2013; Pinto et al. 2015). Birds not only acquire pathogens from the environment, but also return them via excretion, potentially facilitating the dissemination of pathogenic organisms to both humans and other animals, especially through water (Benskin et al. 2009; Wellington et al. 2013). However, further epidemiological studies are necessary to gain a more detailed understanding of the transmission modality of resistant bacteria to wild birds and their spreading into the environment (Guenther et al. 2010; Radhouani et al. 2012).

Of particular concern is the detection of resistance against two molecules belonging to the family of carbapenems, normally used only in human clinical practice as a last resort for treating infections caused by antimicrobial resistant bacteria. It is unclear how wildlife can acquire such resistance. Different hypotheses have been proposed on the phenomenon's genesis, including the great adaptability of bacteria to a variety of environmental displays, their rapid reproduction, the possibility of genetic material exchange among different species and, especially, an intensive use of antibiotics for the treatment of infections both in human and veterinary medicine. Several studies have shown that soil bacteria can represent an important reservoir of antibiotic resistance determinants, including carbapenemases (Gudeta et al. 2015; Nesme and Simonet 2015)

Our results from Passeriformes with an absence of ESBL-producing bacteria are in agreement with other similar studies carried out in Portugal (Silva et al. 2010) and in Sweden (Jarhult et al. 2013). 


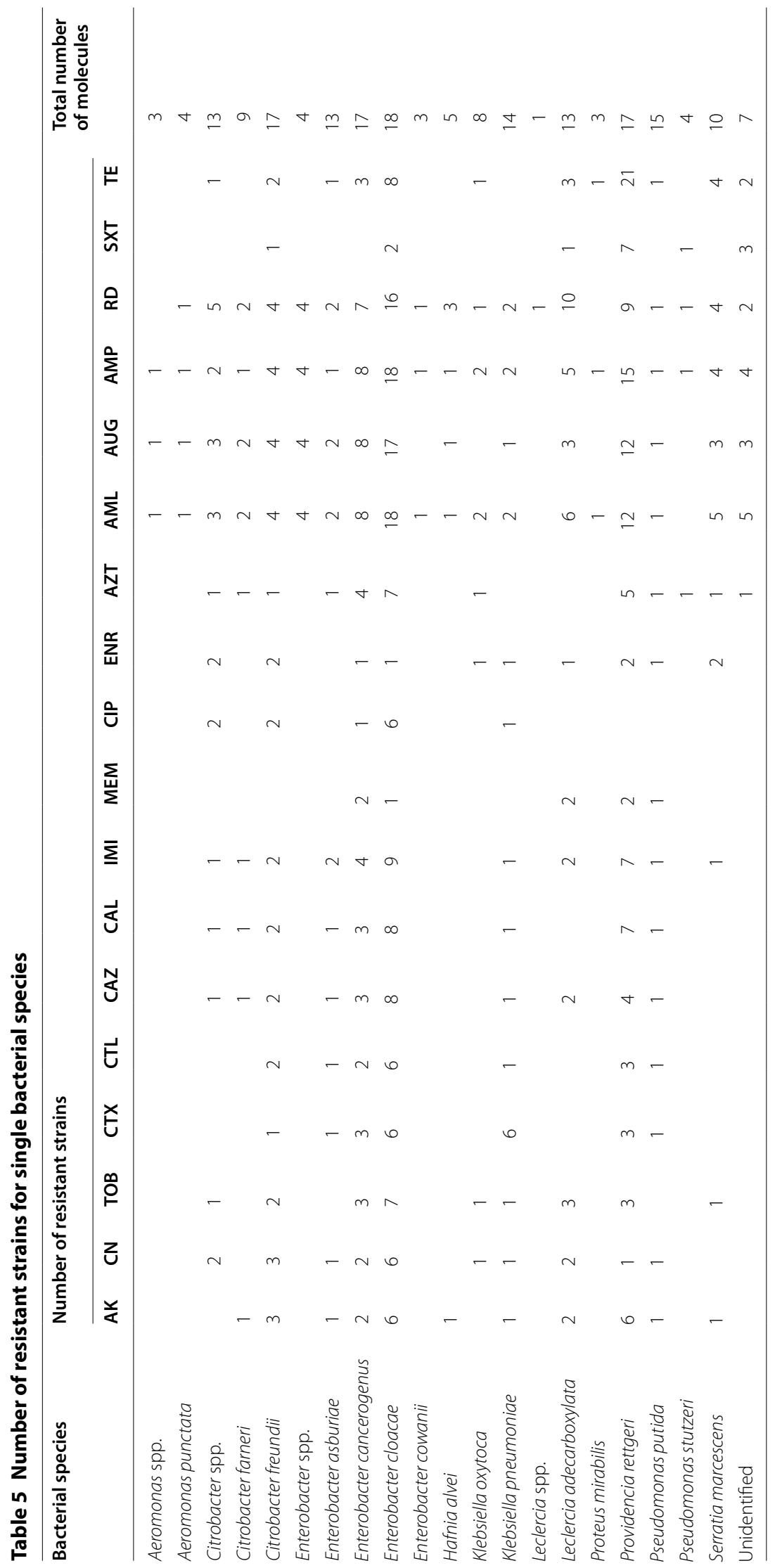


Table 6 Number of molecules against which bacterial strains isolated from each single bird species exhibit resistance

\begin{tabular}{llll}
\hline Family & Bird species & \multicolumn{2}{l}{ Number of molecules } \\
\cline { 3 - 4 } & & Range & Average \\
\hline Emberizidae & Reed Bunting & 1 & $1(5.6 \%)$ \\
Fringillidae & Chaffinch & $4-5$ & $4.5(25 \%)$ \\
Sylviidae & Chiffchaff & $0-15$ & $4(22.2 \%)$ \\
& Firecrest & 4 & $4(22.2 \%)$ \\
Turdidae & Eurasian Blackcap & $0-14$ & $4.8(26.7 \%)$ \\
& European Robin & $0-16$ & $5.3(29.4 \%)$ \\
& Blackbird & $4-9$ & $6.5(36.1 \%)$ \\
& Song Thrush & $3-5$ & $4(22.2 \%)$ \\
\hline
\end{tabular}

\section{Conclusions}

The results of the present study confirmed that migratory wild birds play an important role in the ecology and circulation of potential zoonotic pathogens. Monitoring antibiotic resistance in wildlife represents a useful method of evaluating the impact of anthropic pressure (Thaller et al. 2010). Furthermore, because migratory birds are recognized as potential reservoirs of pathogenic agents, these birds can be regarded as sentinel species and used as environmental health indicators. All these considerations stimulate discussion about the advantages of an integrated monitoring policy of humans, animals and the environment for the antibiotic resistance control (Köck et al. 2017).

\section{Authors' contributions \\ All authors made substantial contributions to conception and design, analysis and interpretation of data. In particular: FM, OBM, FV and LPF also contrib- uted to the laboratory analysis and been involved in drafting the manuscript; $\mathrm{MA}$ and FE also participated in sampling and data collection operations. All authors have read and approved the final manuscript.}

\section{Author details}

${ }^{1}$ Department of Veterinary Sciences, University of Messina, Polo Universitario dell'Annunziata, 98168 Messina, Italy. ${ }^{2}$ Naturalistic Study Milvus, Pantano Street 134, 85010 Pignola, PZ, Italy.

\section{Competing interests}

The authors declare that they have no competing interests.

\section{Availability of data and materials}

The datasets generated and/or analyzed during the current study are available from the corresponding author on reasonable request.

\section{Ethical statement}

All applicable international, national, and institutional guidelines for the care and use of animals were followed. This article does not contain any studies with human participants performed by any of the authors.

\section{Funding}

This research did not receive any specific grant from funding agencies in the public, commercial, or not-for-profit sectors.
Received: 17 May 2017 Accepted: 12 October 2017

Published online: 16 October 2017

\section{References}

Abulreesh H, Goulder R, Scott GW. Wild birds and human pathogens in the context of ringing and migration. Ringing Migr. 2007;23:193-200.

Allen HK, Donato J, Wang HH, Cloud-Hansen KA, Davies J, Handelsman J. Call of the wild: antibiotic resistance genes in natural environments. Nat Rev Microbiol. 2010;8:251-9.

Bangert RL, Ward ACS, Stauber EH, Cho BR, Widders PR. A survey of the aerobic bacteria in the feces of captive raptors. Avian Dis. 1988;32:53-62.

Bauer AW, Kirby WM, Sherris JC, Turck M. Antibiotic susceptibility testing by a standardized single disk method. Am J Clin Pathol. 1966;45:493-6.

Benskin CM, Wilson K, Jones K, Hartley IR. Bacterial pathogens in wild birds: a review of the frequency and effects of infection. Biol Rev. 2009;84:349-73.

Blanco G, Lemus JA, Grande J. Microbial pollution in wild life: linking agricultural manuring and bacterial antibiotic resistance in red-billed choughs. Environ Res. 2009:109:405-12.

Bonnedahl J, Drobni M, Gauthier-Clerc M, Hernandez J, Granholm S, Kayser Y, Melhus A, Kahlmeter G, Waldenstrom J, Johansson A, Olsen B. Dissemination of Escherichia coli with CTX-M Type ESBL between humans and yellow-legged gulls in the South of France. PLoS ONE. 2009:4:e5958.

Brittingham MC, Temple SA, Duncan RM. A survey of the prevalence of selected bacteria in wild birds. J Wildl Dis. 1988;24:299-307.

Camarda A, Circella E, Pennelli D, Madio A, Bruni G, Lagrasta V, Marzano G, Mallia E, Campagnari E. Wild birds as biological indicators of environmental pollution: biotyping and antimicrobial resistance patterns of Escherichia coli isolated from Audouin's gulls (Larus audouinii) living in the Bay of Gallipoli (Italy). Ital J Anim Sci. 2006;5:287-90.

Clinical and Laboratory Standards (CLSI). Performance standards for antimicrobial disk and dilution susceptibility tests for bacteria isolated from animals; approved standard. VET01-A4. 4th Ed. 2013; Wayne, PA, USA.

Elmberg J, Berg C, Lerner H, Waldenström J, Hessel R. Potential disease transmission from wild geese and swans to livestock, poultry and humans: a review of the scientific literature from a one health perspective. Infect Ecol Epidemiol. 2017;7:1300450.

Foti M, Rinaldo D, Guercio A, Giacopello C, Aleo A, De Leo F, Fisichella V, Mammina C. Pathogenic microorganism carried by migratory birds passing through the territory of the Island of Ustica, Sicily (Italy). Avian Pathol. 2011;40:405-9.

Giacopello C, Foti M, Mascetti A, Grosso F, Ricciardi D, Fisichella V, Lo Piccolo F. Antimicrobial resistance patterns of Enterobacteriaceae in European wild bird species admitted in a wildlife rescue centre. Vet Ital. 2016;52:139-44.

Glunder G. Occurrence of Enterobacteriaceae in feces of granivorous passeriform birds. Avian Dis. 1981;25:195-8.

Gudeta DD, Bortolaia V, Amos G, Wellington EM, Brandt KK, Poirel L, Nielsen $J B$, Westh $H$, Guardabassi L. The soil microbiota harbors a diversity of carbapenem-hydrolyzing $\beta$-lactamases of potential clinical relevance. Antimicrob Agents Chemother. 2015;60:151-60.

Guenther S, Grobbel M, Lubke-Becker A, Goedecke A, Friedrich ND, Wieler LH, Ewers C. Antimicrobial resistance profiles of Escherichia coli from common European wild bird species. Vet Microbial. 2010;144:219-25.

Hubálek Z. An annotated checklist of pathogenic microorganisms associated with migratory birds. J Wild Dis. 2004;40:639-59.

Jarhult JD, Stedt J, Gustafsson L. Zero prevalence of extend spectrum betalactamase-producing bacteria in 300 breeding Collared Flycatchers in Sweden. Infect Ecol Epidemiol. 2013;3:20909.

Köck R, Kreienbrock L, van Duijkeren E, Schwarz S. Antimicrobial resistance at the interface of human and veterinary medicine. Vet Microbiol. 2017;200:1-5.

Liofilchem ${ }^{\circledR}$. Antibiotic disc interpretative criteria and quality control—Rev.12. 2016. http://www.liofilchem.net/pdf/disc/disc_interpretative_table.pdf. Accessed 30 Jun 2016.

Literak I, Dolejska M, Janoszowska D, Hrusakova J, Meissner W, Rzyska H, Bzoma S. Cizek A Antibiotic resistant Escherichia coli bacteria, including strains with genes encoding the extended-spectrum beta-lactamase and QnrS, in waterbirds on the Baltic Sea Coast of Poland. Appl Environ Microbiol. 2010;76:8126-34 
Magda AM, Mohamad NM, Maysa Al, Merwad AM, Rasha MA, Rasha MM, Rehab E. Prevalence of Enterobacteriaceae in wild birds and humans at Sharkia Province; With special reference to the genetic relationship between E.coli and Salmonella isolates determined by protein profile analysis. J Am Sci. 2013;9:173-83.

Matias CA, Pereira IA, Reis EM, Rodrigues DD, Siciliano S. Frequency of zoonotic bacteria among illegally traded wild birds in Rio de Janeiro. Braz J Microbiol. 2016;47:882-8.

Nesme J, Simonet P. The soil resistome: a critical review on antibiotic resistance origins, ecology and dissemination potential in telluric bacteria. Environ Microbiol. 2015;17:913-30.

Pindi PK, Yadav PR, Shanker AS. Identification of opportunistic pathogenic bacteria in drinking water samples of different rural health and their clinical impacts on humans. Biomed Res Int. 2013. doi:10.1155/2013/348250.

Pinto A, Simões R, Oliveira M, Vaz-Pires P, Brandão R, da Costa PM. Multidrug resistance in wild bird populations: importance of the food chain. J Zoo Wildl Med. 2015;46:723-31.

Radhouani H, Poeta P, Gonçalves A, Pacheco R, Sargo R, Igrejas G. Wild birds as biological indicators of environmental pollution: antimicrobial resistance patterns of Escherichia coli and enterococci isolated from common buzzards (Buteo Buteo). J Med Microbiol. 2012;61:837-43.

Reed KD, Meece JK, Henkel JS, Shukla SK. Birds, migration and emerging zoonoses: west Nile virus, Lyme disease, influenza A and enteropathogens. Clin Med Res. 2003;1:5-12.

Reslinski A, Gospodarek E, Mikucka A. Prevalence of multidrug-resistant Proteus spp. strains in clinical specimens and their susceptibility to antibiotics. Med Dosw Mikrobiol. 2005;57:175-84.
Schwarz S, Silley P, Simjee S, Woodford N, van Duijkeren E, Johnson AP, Gaastra W. Editorial: assessing the antimicrobial susceptibility of bacteria obtained from animals. Vet Microbiol. 2010;65:601-4.

Shobrak MY, Abo-Amer AE. Role of wild birds as carriers of multi-drug resistant Escherichia coli and Escherichia vulneris. Braz J Microbiol. 2015;45:1199-209.

Silva N, Felgar A, Goncalves A, Correia S, Pacheco R, Araújo C, Igrejas G, Poeta P. Absence of extended-spectrum-betalactamase-producing Escherichia coli isolates in migratory birds: song thrush (Turdus philomelos). J Antimicrob Chemother. 2010;65:1306-7.

Skurnik D, Ruimy R, Andremont A, Amorin C, Rouquet P, Picard B, Denamur E. Effect of human vicinity on antimicrobial resistance and integrons in animal faecal Escherichia coli. J Antimicrob Chemother. 2006;57:1215-9.

Steele CM, Brown RN, Botzler RG. Prevalences of zoonotic bacteria among seabirds in rehabilitation centers along the Pacific Coast of California and Washington, USA. J Wildl Dis. 2005;41:735-44.

Thaller MC, Migliore L, Marquez C, Tapia W, Cedeno V, Rossolini GM, Gentile G. Tracking acquired antibiotic resistance in commensal bacteria of Galapagos land iguanas: no man, no resistance. PLoS ONE. 2010;5:e8989.

Wellington EM, Boxall AB, Cross P, Feil EJ, Gaze WH, Hawkey PM, JohnsonRollings AS, Jones DL, Lee NM, Otten W, Thomas CM, Williams AP. The role of the natural environment in the emergence of antibiotic resistance in gram-negative bacteria. Lancet Infect Dis. 2013;13:155-65.

\section{Submit your next manuscript to BioMed Central and we will help you at every step:}

- We accept pre-submission inquiries

- Our selector tool helps you to find the most relevant journal

- We provide round the clock customer support

- Convenient online submission

- Thorough peer review

- Inclusion in PubMed and all major indexing services

- Maximum visibility for your research

Submit your manuscript at www.biomedcentral.com/submit
() Biomed Central 